\title{
HOW GOOD ARE THE NEW COMPUTERS?
}

\author{
Chess Computer News \\ Special Edition and February 1987 Supplement \\ ISSN 0263-7464
}

We quote the introduction:

This special edition of chess computer news is justified firstly by the breakthrough made by Dan and Kathe Spracklen and the Fidelity International programming team, and secondly and most importantly, by the fact that an International Grandmaster of the stature of Jon Speelman was sufficiently impressed by the standard of play to be willing to write very detailed, highly readable, and very informative annotations to six of the 33 games (three wins, two draws, and a loss) - providing players of all standards, from beginners to Grandmaster, with an invaluable, independent, and balanced guide to this new standard of computer play.

We have long maintained that the most reliable way to determine the true standard of a computer's play is to have it play a minimum of $\mathbf{3 0}$ games against strong players under the strict conditions of an official tournament - and for it to thus earn an appropriate Official Chess Federation grading.

Jon Speelman:" In annotating the six games selected I've tried to give an impartial account of both Par Excellence's strenghts and its weakness, and also to provide notes wich will be of interest to a wide range of chessplaying abilities. I hope that I haven't failed too badly in either respect....."

\section{CORRECTION}

by The Editor

To our great regret, in the previous issue the bottom line was missing from page 176 in the article "Fuzzy Production Rules in Chess" by Prof. dr. P.W. Frey. We hasten to correct the error, reprinting below the missing line (marked by bars) in its context.

In a recent article in BYTE magazine (Frey, 1986), I described an atypical expert system for making categorization judgments. The technique involves using many bit-maps to code information about specific patterns and a single bit-map to code the characteristics of the current board position. Boolean computer | operations are then employed to measure the similarity between the current situation and each of the | specific patterns stored in the database. This approach does not require an exact match. Instead, the amount of evidence consistent with each pattern is assessed and the action side of the production rule is implemented only when the weight of evidence exceeds a predetermined threshold. This technique involves descriptive characteristics of three varieties. 